${ }^{1}$ Affiliation not available

January 4, 2021

Happiness Optimizer: a swarm intelligence algorithm for finding global minimum

Aref Yelghi

Aref.yelghi@avrasya.edu.tr

Avrasya University, Faculty of Engineering and Architecture, Computer Engineering, Trabzon, Yomra, 61250

Shirmohammad Tavangari

s.tavangari@alumni.ubc . ca

Electrical and Computer Engineering, 2332 Main Mall, Vancouver, BC Canada V6T 1Z4

\title{
ABSTRACT
}

Recent work attempted to demonstrate the global best minimum in complex problems. This paper proposes a population and direct-based swarm optimization algorithm called as HPO algorithm. The HPO algorithm is designed by inspired of personal behaviorand demonstrated in the 30 and 100 dimensionson benchmark functions. The model have four concepts: what you want?, what you have?, what others have?, what is happened?. These concepts take into account the balancing between exploration and exploitation operator and demonstrate its efficiency, robustness and stability insynthetic and real problems.In experiment, we consider 15 benchmark functions include unimodal and multimodal characteristic of functions.For compression, our algorithm and some well-known algorithms with 30 times run and applied on the benchmark functions and compared with statistical value and Wilcoxon signed-rank test. As a consequence, the performance and convenient ofour work aredemonstratedbetter than the others.

\section{1- INTRUDOCTION}

Many real-world applications include the complexity problem that should be optimize and then applied on the real work. The purpose of optimization is the minimization or maximization of fitness function for real problem such as energy consumption, designing, routing, transportation et al. The performance, efficiency and sustainability of optimization algorithms are important in solving complex problem.

In many case, optimization problem is highly complex and nonlinear function, whom scientist attempts to solve the problem by using popular methods of soft computing. In recent years, metaheuristic algorithms have been used and applied on the real problems in engineering field [1-5]. The advantage of metaheuristic algorithm rather than the deterministic algorithm is scape of trap from local minimum state. There are two popular methodology such as Evolutionary Algorithms (EA) and Swarm Intelligence (SI), which have new landscape for the complex problem in the metaheuristics optimization algorithm. The advantages of them are the power of based-population solution and providea new solutionby considering the balance of exploration and exploitation. Thepoint of view of efficiency and balancing of them should be se the best solution by escaping from many local minimum.By inspired of Darwin's theory the Evolutionary Optimization techniques are presented and used in real problems. The principle of mechanism includes selection, mutation and crossover operation. Genetic algorithm is one of EA which is popular in metaheuristic algorithm (GA) and have rigorous mathematical analyses [6-7].And some works with based on this paradigm is tabu 
search [9], simulated annealing [10], forest optimization algorithm [11], biogeography-based optimizer (BBO) [12], Evolutionary Programing (EP) [13], Evolution Strategy (ES) [14].Beni and Wang in 1993 presented concept of swarm intelligence, which include simulation of behavior of living creature [15]. Scientist attempt to find local rule between creatures and then convert to aalgorithm for using in soft computing. The other algorithmsare computational method, which based on directed best agent. The framework of them are repeatedlytrying to improve a solution in relation toa given measure of quality fitness.Examples of SI-based approaches are particle swarm optimization [16], Glowworm algorithm [17] Intelligent water drops [18], Cat Swarm Optimization [19], artificial bee colony(ABC) [20], Gravitational search algorithm [21] and selfish herdoptimizer (SHO) [22], Dolphin Echolocation (DE) [23].Some algorithms inspired by the phenomenon of physics are proposed and surveyed for example Central Force Optimization CFO[24], Artificial Physics Optimization APO[25], Gravitational Search Algorithm GSA[26], Gravitational Interactions Optimization GIO[27]. The No Free Lunch (NFL) theorem logically proved that there is no metaheuristic algorithms capable to solve the general problem.In order to improve the flexible of optimization algorithm for solving more problems scientist attempt to present novel algorithm or improve the old version of algorithms, which are able to solve general problems [28]. There are many algorithms proposed which have advantages and disadvantages. In this study,mathematical analyzing, demonstratingthe convergenceof ,large-scale problems and tuning parameters are considered and provided a novel method for solving optimization problems.

The restof the paper is organized as follows. Section 2 provides the detail of HPO algorithm and discusses about the concept of exploration and exploitation. The experimental results and evaluation are shown in Section 3. Section 4 provides the performance of HPO on a real problem. Section 5 states some concluding remarks and suggests some directions for future.

\section{2- HAPPINESS OPTIMIZER}

As discussed in book[29]general equation is that "Happiness equals Reality minus Shifting Expectations," and indicate that happiness is always on the move and difficult to find, whilethe expectations follow reality. To preserve happiness in the mind, one needs to achieve control on the expectations and assure reality is one-steppast. As a result, when the reality of Human beings' life is better than they had expected, theywould be happiness in their life. Otherwise reality to be worse than the expectations, they would be unhappiness. The other words, when you think about high expectation you will be face with negative realization slit, which means more exposed to unhappiness in the future. Therefore, we can take general equation and which is discussed about it in [30-31].In this study, a new population-based algorithmcalled as Happiness Optimizer (HPO)isproposed,that inspired by the theory ofHappiness in social science field. Four main concepts of the Happiness theory (what you want, what is happen, what you have, and what others have) are mathematically modelled to build the HPO.As mentioned before the formula is Eq (1).

Happiness= Reality minus Expectation (1)

Based on the Equation 1 there arefour effective component one is for reality and remain is for the expectation such as:

1. What is have

2. What is happen

3. What you want

4. What others have

As mentioned above, our expectation are infinitive. They are function of outdoor event, "what you want" in short and long time and "what others have" in neighbors(such as workplace, neighbors and etc). "What is happen "is related to a thing that happens in neighbors or the world which are important for you (see Fig $1)$. 

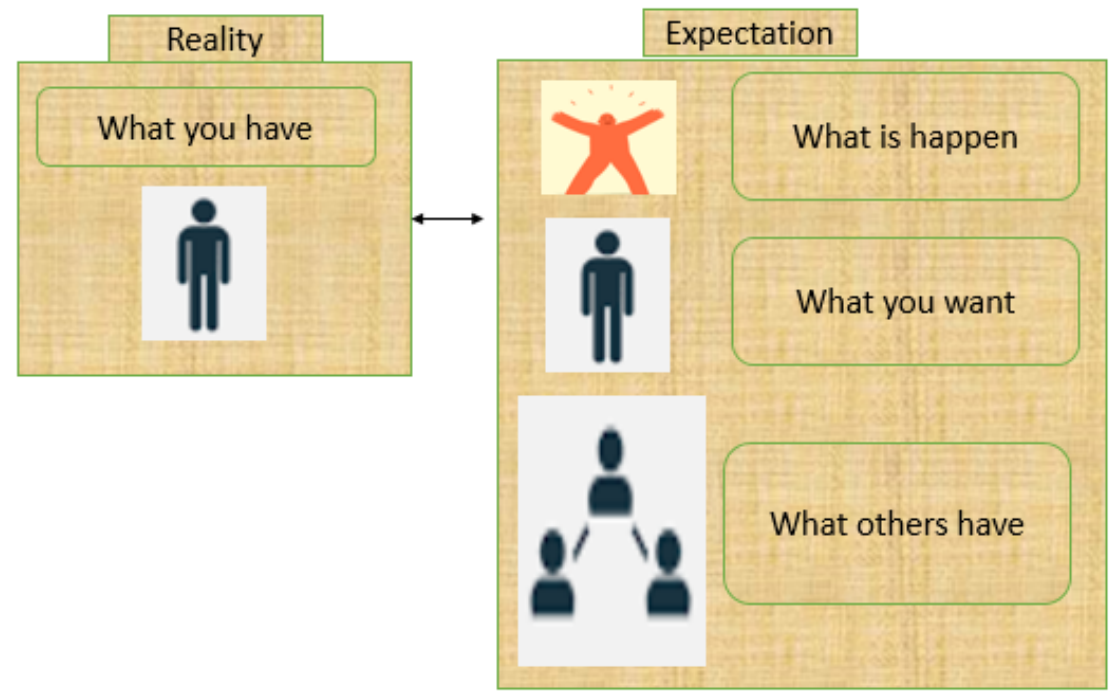

Fig 1.Happiness Model

\section{2-1 INSPIRATION}

In a company, some personals attempt to obtain a new positon instead of old position, which leads causes to be happiness. By changing, the behavior of personal in the company we can modeled to an algorithm, which can be one of the specific branch of swarm intelligence.

The model by four behaviors, which mentioned before, can be make decision in the space of problem. The model should take into account the balancing exploration and exploitation in the space of problems. Four agent based on mentioned behaviorare indicated and introduced as follows

Ps $\mathrm{H}=$ the history of personal

Ps $\mathrm{C}=$ the current personal

Ps $N_{1}=$ the best neighbor of current personal

Ps $G=$ the best personal in company or companies

The cost valuesof eachagentsare converted to the fitness value, which is defined in general equation 2. After computing, we calculate probability value for each agent, whichis given in the equation3.

Fitness $_{i}=\operatorname{Exp}\left(\frac{f_{i}}{\operatorname{Average}(F)}\right) \mathrm{F}=\left\{f_{1} \ldots f_{i} \ldots f_{n}\right\} \mathrm{n}=4(2)$

$P_{i}=\frac{\mathrm{Fit}_{i}}{\sum_{i=1}^{n} \mathrm{Fit}_{i}}(3)$

The space of movement agents determined with twocriterionfor balancing the exploration and exploitation and scape of local minimum.

First criterion:

If the cost value of the first neighbor is less than the cost value ofthe current agentsatisfied it leads to two statesbased on the generated uniformvalue including coefficient formula. Rr1: uniformly distributed random number in the interval $(0,1)$ (what is happen in the world) L: current iteration n: number of agents $\mathrm{pn}=\mathrm{n}^{*} 0.75 \mathrm{Sum}=\sum_{i=1}^{\mathrm{pn}} p_{i}(4) \mu_{1}=2-\mathrm{L}^{*}((2) /$ iteration number $)(5) \mu_{2}=1-\mathrm{L}^{*}((1) /$ iteration number $)$ $(6) \varnothing=1-\mathrm{L}^{*}((1) /$ iteration number $)(7)$ alpha $=0.6-\mathrm{I}^{*}((0.6-0.09) /$ iteration number $)(8)$

\section{1)}


$\operatorname{Rr} 1<\varnothing$

$H_{i, j_{\text {new }}}=H_{i, j_{\text {old }}} \times$ alpha $+\mu_{1} \times \operatorname{Sum} * \operatorname{Rand}(0,1) \times\left(x_{j_{\mathrm{GB}}}-x_{i, j}\right)+\mu_{2} \times p_{2} \times\left(x_{i, j_{p_{2}}}-x_{i, j}\right)(9)$

\section{2)}

$\operatorname{Rr} 1>\varnothing$

Delta $_{i, j}=x_{j \mathrm{~GB}}$

This criteria with two statesbased on the growth generation is provided. In state number 1.1 we take into account the path of other agents such as (what others have and what you want) presented a balancing between the exploration and exploitation in the space which include four damping equation with tuned parameters (Equations 5,6,7,8). The other side state number 1.2 by passing the generation to the next generation,gradually it switch to exploitation operation with only simulate the behavior of global best.

Second criterion:

2) If the first criterionis not satisfied, the second criterion will perform with two status.

\section{1)}

$\operatorname{Rr} 2<\varnothing$

Delta $_{i, j}=$ alpha $^{*} \operatorname{Delta}_{i, j}+Q_{1} *\left(x_{j \mathrm{~GB}}-x_{i, j}\right)$

2.2)

$\operatorname{Rr} 2>\varnothing$

Delta $_{i, j}=$ alpha $^{*} \operatorname{Delta}_{i, j}+Q_{2} *\left(x_{j \mathrm{~GB}}-x_{i, j}\right)$

The equation 11 and 12are definedin order to scape of local minimum and provide the deviation ofthe current position.

Totally, two mentioned abovecreation with three status of new positon are indicated in Fig 2,3 and 4.In figure $x_{1}, x_{2}$ are the positon of agents in two dimensional of problem. General formula for our model (what you have) is defined in the equation 13. The algorithm HPO is presented in the Fig 5.

$x_{\text {new } i, j}=x_{\text {old } i, j}+$ Delta $_{i, j}(13)$

Totally, this method is specified type of PSO algorithm. Where, by inspired of happiness model the searching space is divided to three spaces, which is more suitable for balancing searching and more power in the get rid of local minimum.

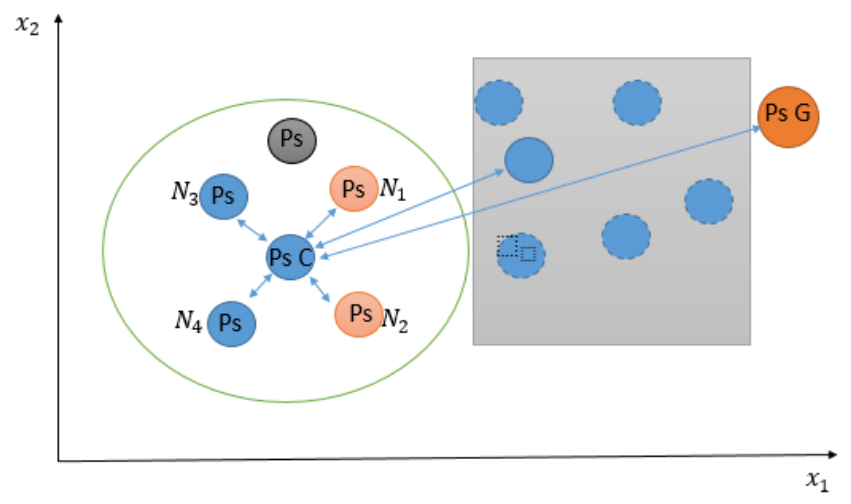

Ps $\mathrm{H}=P_{1}$ Grey Color

Ps $\mathrm{C}=P_{2}$

Ps $N_{1}=P_{3}$

Ps $G=P_{4}$

Fig 2.New positon in inside of the grey square with equation 9 


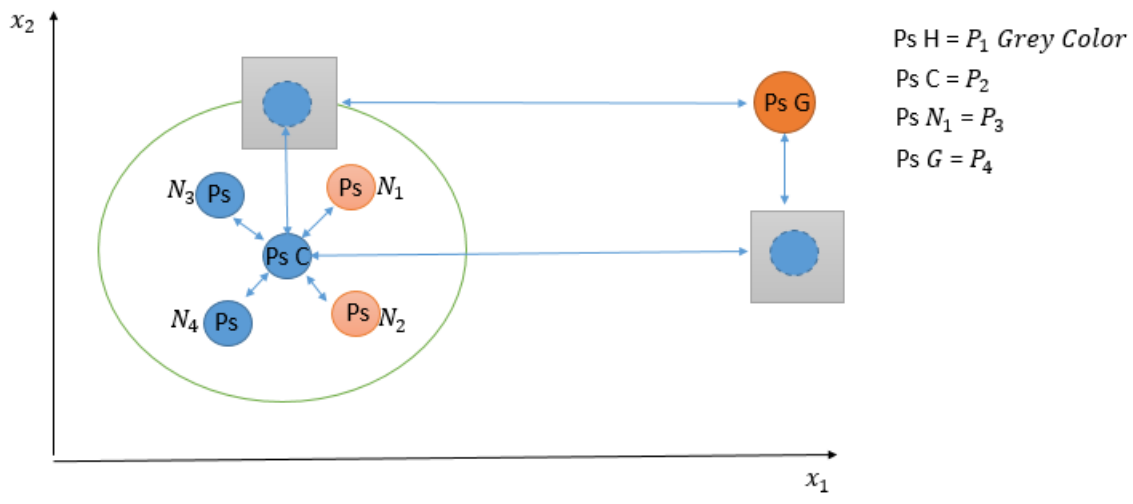

Fig 3. New positon in inside of the grey square with equation 10

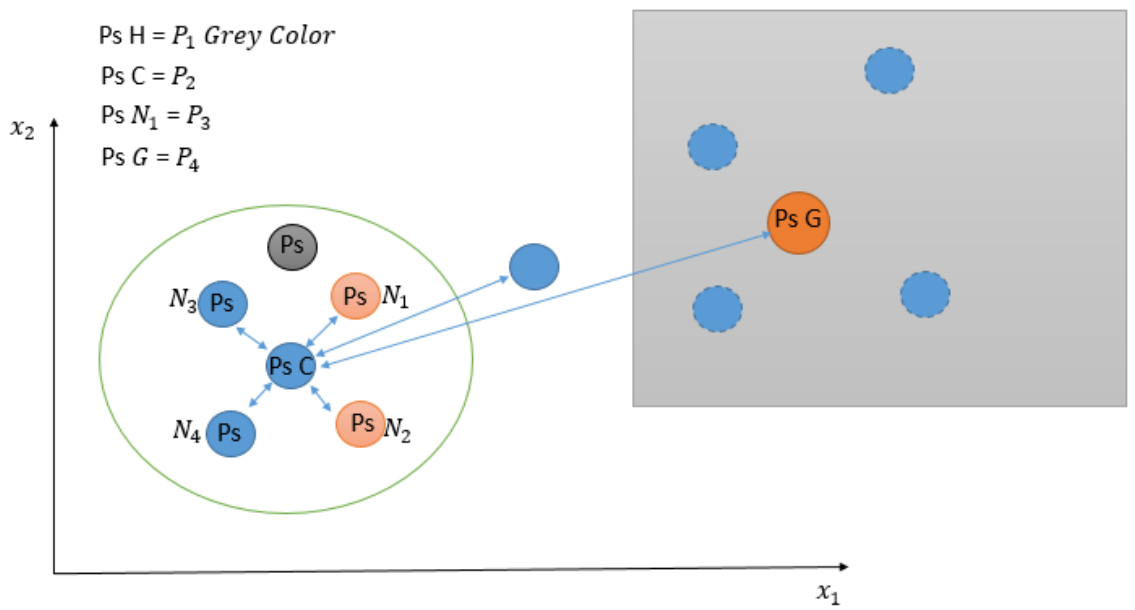

Fig 4.New positon inthe inside of the grey square with equation 11 and 12

Fig 5.HPO Algorithm

ComparativeStudy We statistically compared the HPO algorithm with GWO, PSO, ABC and FA algorithms to demonstrate itsefficiency, robustnessand stability for both 30 and 100 dimensionsin benchmark functions. In experiment, we consider 15 benchmark functions include unimodal and multimodal functions for comparing that showed in Tables 1 and 2 and visualize in Fig 6.

\begin{tabular}{llll}
\hline F1 & F2 & F3 & F4 \\
\hline F5 & F6 & F7 & F8 \\
F9 & F10 & F11 & F12 \\
F13 & F14 & F15 & \\
\hline
\end{tabular}

Fig 6. Search space of UCI benchmark function

4.1 Statistical discussion

In each experiment, all algorithms executed 30 timesand each running performed with 500 iterations for 
all benchmark functions.From the other side, this comparison is not enough for reliability. Furthermore, we applied the Wilcoxon Signed-Rank test, which provide statistically validate in the results. The test is performed using a pairwise method, where $\mathrm{p}$ and his the significance and logic valuerespectively,that based on whether the defined hypothesis is rejected or accepted. Test including the following hypothesis

$\mathrm{a}=0.05(95 \%)$

$H_{0}: \mu_{1}=\mu_{2}$

$H_{1}: \mu_{1} \neq \mu_{2}$

Two algorithm with the obtained results are compared and considered their valuesare near or faraway $\left(H_{0}\right.$ and $H_{1}$ ) to each other.

$H_{0}=0$ two algorithm are not different.

$H_{1}=1$ two algorithm are different.

In according with the outcome of Tables 3,4,5,6. We observed our algorithm surpasses than the others in balancing exploitation and exploration and finding global minimum. To sum up, our algorithm with competitive result showed the power on the unimodal and multimodal benchmark functions in complex problem. One example of divergence for all algorithmsshowon the Sphere function in Fig 7. As you view, HPO in less iteration has not proper diversity, but in more dimensionsHPO algorithm has appropriate diversity and result than the others.

\subsection{Real Problem}

For further examine, the performance of HPO, a real problem employed in the function, pressure vessel designs,problem which is constrained engineering design,are used.HPOalgorithm should have a constraint handling strategy to optimize the constrained problem. Considering the equality and non-equality constrain in the problem,algorithms should control the constraintand object value with considering violence. The objective of pressure vessel designs is to minimize the total cost with constraint comprising of welding, forming, and material of a cylindrical vessel. Four design process factors or four decision variable should be tuned by algorithms which is mentioned as follows.

- Thickness of the shell $(\mathrm{Ts})$.

- Thickness of the head (Th).

- Inner radius (R).

- Length of the cylindrical portion without regard to the head

(L).

The objective function and four constraint functions aredefined in Equation 14.An expression of the Pressure Vessel Design (PVD) is as follows: both ends of a cylindrical vessel are capped by hemispherical heads (Fig 8).

Consider $\vec{x}=\left[x_{1} x_{2} x_{3} x_{4}\right]=\left[T_{s} T_{h} \mathrm{RL}\right]$,

Minimize $\mathrm{f}(\vec{x})=0.6224 x_{1} x_{2} x_{3}+1.7781 x_{2} x_{3}^{2}+3.1661 x_{1}^{2} x_{4}+19.84 x_{1}^{2} x_{3}$,

Subject to $g_{1}(\vec{x})=-x_{1}+0.0193 x_{3} \leq 0$,

$g_{2}(\vec{x})=-x_{3}+0.0954 x_{3} \leq 0$,

$g_{3}(\vec{x})=-\pi x_{3}^{2} x_{4}-\frac{4}{3} \pi x_{3}^{3}+1296000 \leq 0$,

$g_{4}(\vec{x})=-x_{4}-240 \leq 0,(14$

The upper and lower decision variablesare defined in the following Eq. 15 
$0 \leq x_{1} \leq 99$

$0 \leq x_{2} \leq 99$,

$10 \leq x_{3} \leq 200$

$10 \leq x_{4} \leq 200(15$

In Table 7. Comparing all algorithmin vessel pressure design optimization, which include tuned parameters and cost value. As you view, the performance of our algorithm take constraint challenge into consideration outperforms the others.

Table 1. Unimodalbenchmarkfunctions

\begin{tabular}{llll}
\hline Function name & Dimension & Range & $f_{\text {min }}$ \\
\hline$f_{1}(x)=\sum_{i=1}^{n} x_{i}^{2}$ & 50,100 & {$[-100,100]$} & 0 \\
$f_{2}(x)=\sum_{i=1}^{n}\left|x_{i}\right|+\prod_{i=1}^{n}\left|x_{i}\right|$ & 50,100 & {$[-10,10]$} & 0 \\
$f_{3}(x)=\sum_{i=1}^{n}\left(\sum_{j-1}^{i} x_{j}\right)^{2}$ & 50,100 & {$[-100,100]$} & 0 \\
$f_{4}(x)=\max _{i}\left\{\left|x_{i}\right|, 1 \leq i \leq n\right\}$ & 50,100 & {$[-100,100]$} & 0 \\
$f_{5}(x)=\sum_{i=1}^{n-1}\left[100\left(x_{i+1}-x_{i}^{2}\right)^{2}+\left(x_{i}-1\right)^{2}\right]$ & 50,100 & {$[-30,30]$} & 0 \\
$f_{6}(x)=\sum_{i=1}^{n}\left(\left[x_{i}+0.5\right]\right)^{2}$ & 50,100 & {$[-100,100]$} & 0 \\
$f_{7}(x)=\sum_{i=1}^{n} \mathrm{ix}_{i}^{4}+\operatorname{random}(0,1)$ & 50,100 & {$[-1.28,1.28]$} & 0 \\
\hline
\end{tabular}

Table2. Multimodal benchmark functions

\begin{tabular}{|c|c|c|c|}
\hline Function name & Dimension & Range & $f_{\min }$ \\
\hline \multicolumn{4}{|c|}{$\sum_{i=1}^{n}-x_{i} \sin \left(\sqrt{\left|x_{i}\right|}\right)$} \\
\hline $\begin{array}{l}f_{9}(x)= \\
\sum_{i=1}^{n}\left[x_{i}^{2}-10 \mathrm{co}\right.\end{array}$ & \multicolumn{2}{|c|}{$\sum_{i=1}^{n}\left[x_{i}^{2}-10 \cos \left(2 \pi x_{i}\right)+10\right]$} & 0 \\
\hline \multicolumn{4}{|c|}{$\begin{array}{l}-20 \exp \left(-0.2 \sqrt{\frac{1}{n} \sum_{i=1}^{n} x_{i}^{2}}\right)- \\
\exp \left(\frac{1}{n} \sum_{i=1}^{n} \cos \left(2 \pi x_{i}\right)\right)+ \\
20+e\end{array}$} \\
\hline \multicolumn{4}{|c|}{$\frac{1}{4000} \sum_{i=1}^{n} \cos \left(\frac{x_{i}}{\sqrt{i}}\right)+1$} \\
\hline & 30,100 & {$[-50,50]$} & 0 \\
\hline \multicolumn{4}{|c|}{$f_{12}(x)=\frac{\pi}{n}\left\{10 \sin \left(\pi y_{1}\right)+\sum_{i=1}^{n-1}\left(y_{i}-1\right)^{2}\left[1+10 \sin ^{2}\left(\pi y_{i+1}\right)\right]+\left(y_{n}-1\right)^{2}\right\}+\sum_{i=1}^{n} u\left(x_{i}, 10,100,4\right)$} \\
\hline \multicolumn{4}{|c|}{$\begin{array}{l}y_{i}=1+\frac{x_{i}+1}{4} \\
f_{13}(x)=0.1\left\{\sin ^{2}\left(3 \pi x_{1}\right)+\sum_{i}^{3} \underline{\underline{0}}_{1} 1\left(00_{i}-1\right)^{2}\left[1+\sin ^{2}\left(3 \pi x_{i}+50\right)_{j} 5 \theta\right]\left(x_{n}-1\right)^{2}\left[1+\sin ^{2}\left(2 \theta x_{n}\right)\right]\right\}+ \\
\sum_{i=1}^{n} u\left(x_{i}, 5,100,4\right)\end{array}$} \\
\hline $\begin{array}{l}f_{14}(x)= \\
{\left[e^{-\sum_{i=1}^{n}\left(\frac{x_{i}}{\beta}\right)^{2 m}}\right.}\end{array}$ & $\begin{array}{l}30,100 \\
\prod_{i}^{n} \cos ^{2} x\end{array}$ & {$[-20,20]$} & -1 \\
\hline
\end{tabular}




\begin{tabular}{|c|c|c|c|}
\hline Function name & Dimension & Range & $f_{\min }$ \\
\hline$f_{15}(x)=\left\{\left[\sum_{i=}^{n}\right.\right.$ & Beppl $\left.1\left(0 \theta \sum_{i=1}^{n} x^{2}\right)\right\} \cdot \exp$ & $\left.\left.-\sum_{i}^{n}=1_{1} 0 \sin \theta^{2}\right] \sqrt{\left|x_{i}\right|}\right]$ & -1 \\
\hline
\end{tabular}

Table 3.Result of unimodal and multimodal benchmark function in 30 dimensions

\begin{tabular}{|c|c|c|c|c|c|c|}
\hline Function & Statitic value & GOW & $\mathrm{PSO}$ & HPO & $\mathrm{ABC}$ & $\mathrm{FA}$ \\
\hline \multirow[t]{3}{*}{$\overline{\mathrm{F} 1}$} & minimum & $5.12 \mathrm{E}-29$ & $4.45 \mathrm{E}+01$ & $1.07 \mathrm{E}-41$ & $4.14 \mathrm{E}+01$ & 8.09E-08 \\
\hline & avrage & $1.24 \mathrm{E}-27$ & $7.20 \mathrm{E}+02$ & $6.81 \mathrm{E}-37$ & $1.46 \mathrm{E}+02$ & $1.11 \mathrm{E}-07$ \\
\hline & std & $2.83 \mathrm{E}-54$ & $1.69 \mathrm{E}+05$ & $5.34 \mathrm{E}-72$ & $8.86 \mathrm{E}+03$ & $2.08 \mathrm{E}-16$ \\
\hline \multirow[t]{3}{*}{$\mathrm{F} 2$} & minimum & $1.49 \mathrm{E}-17$ & $6.27 \mathrm{E}-01$ & $1.90 \mathrm{E}-22$ & $3.28 \mathrm{E}+00$ & $1.16 \mathrm{E}-04$ \\
\hline & avrage & $9.61 \mathrm{E}-17$ & $7.38 \mathrm{E}+00$ & $5.01 \mathrm{E}-20$ & $5.57 \mathrm{E}+01$ & $1.40 \mathrm{E}-04$ \\
\hline & std & $3.73 \mathrm{E}-33$ & $1.89 \mathrm{E}+01$ & $2.00 \mathrm{E}-38$ & $8.70 \mathrm{E}+02$ & $9.13 \mathrm{E}-11$ \\
\hline \multirow[t]{3}{*}{ F3 } & minimum & $2.49 \mathrm{E}-08$ & $3.57 \mathrm{E}+01$ & $9.57 \mathrm{E}-33$ & $3.71 \mathrm{E}+04$ & $5.24 \mathrm{E}-05$ \\
\hline & avrage & $7.74 \mathrm{E}-06$ & $1.84 \mathrm{E}+03$ & $1.00 \mathrm{E}-26$ & $6.94 \mathrm{E}+04$ & $1.47 \mathrm{E}-03$ \\
\hline & std & $8.92 \mathrm{E}-11$ & $1.01 \mathrm{E}+07$ & $2.51 \mathrm{E}-51$ & $2.11 \mathrm{E}+08$ & $4.79 \mathrm{E}-06$ \\
\hline \multirow[t]{3}{*}{$\mathrm{F} 4$} & minimum & $5.44 \mathrm{E}-08$ & $1.43 \mathrm{E}+00$ & $6.32 \mathrm{E}-19$ & $5.70 \mathrm{E}+01$ & $1.44 \mathrm{E}-04$ \\
\hline & avrage & $9.10 \mathrm{E}-07$ & $7.29 \mathrm{E}+00$ & $1.27 \mathrm{E}-16$ & $6.41 \mathrm{E}+01$ & $1.57 \mathrm{E}+00$ \\
\hline & std & $2.02 \mathrm{E}-12$ & $3.64 \mathrm{E}+01$ & $9.65 \mathrm{E}-32$ & $1.50 \mathrm{E}+01$ & $3.23 \mathrm{E}+00$ \\
\hline \multirow[t]{3}{*}{ F5 } & minimum & $2.61 \mathrm{E}+01$ & $1.37 \mathrm{E}+02$ & $2.61 \mathrm{E}+01$ & $7.55 \mathrm{E}+05$ & $2.28 \mathrm{E}+01$ \\
\hline & avrage & $2.71 \mathrm{E}+01$ & $4.63 \mathrm{E}+04$ & $3.05 \mathrm{E}+01$ & $2.86 \mathrm{E}+06$ & $3.19 \mathrm{E}+01$ \\
\hline & std & $5.88 \mathrm{E}-01$ & $5.39 \mathrm{E}+09$ & $2.66 \mathrm{E}+02$ & $1.86 \mathrm{E}+12$ & $3.47 \mathrm{E}+02$ \\
\hline \multirow[t]{3}{*}{ F6 } & minimum & $2.55 \mathrm{E}-01$ & $1.61 \mathrm{E}+02$ & $5.30 \mathrm{E}-01$ & $2.00 \mathrm{E}+01$ & $7.96 \mathrm{E}-08$ \\
\hline & avrage & 7.93E-01 & $6.80 \mathrm{E}+02$ & $1.20 \mathrm{E}+00$ & $1.43 \mathrm{E}+02$ & $1.08 \mathrm{E}-07$ \\
\hline & std & $9.04 \mathrm{E}-02$ & $1.80 \mathrm{E}+05$ & $1.45 \mathrm{E}-01$ & $8.60 \mathrm{E}+03$ & $1.35 \mathrm{E}-16$ \\
\hline \multirow[t]{3}{*}{ F7 } & minimum & $2.64 \mathrm{E}-04$ & $3.55 \mathrm{E}-02$ & $6.23 \mathrm{E}-05$ & 8.80E-01 & $2.05 \mathrm{E}-03$ \\
\hline & avrage & $1.61 \mathrm{E}-03$ & $1.27 \mathrm{E}-01$ & $1.06 \mathrm{E}-03$ & $1.54 \mathrm{E}+00$ & $4.22 \mathrm{E}-03$ \\
\hline & std & $8.36 \mathrm{E}-07$ & $2.97 \mathrm{E}-03$ & $7.72 \mathrm{E}-07$ & 4.33E-01 & $2.41 \mathrm{E}-06$ \\
\hline \multirow[t]{3}{*}{ F8 } & minimum & $-7.89 \mathrm{E}+03$ & $-3.71 \mathrm{E}+03$ & $-1.19 \mathrm{E}+04$ & $-2.22 \mathrm{E}+61$ & $-1.03 \mathrm{E}+04$ \\
\hline & avrage & $-6.28 \mathrm{E}+03$ & $-3.01 \mathrm{E}+03$ & $-7.83 \mathrm{E}+03$ & $-1.66 \mathrm{E}+60$ & $-8.95 \mathrm{E}+03$ \\
\hline & std & $7.87 \mathrm{E}+05$ & $1.66 \mathrm{E}+05$ & $3.71 \mathrm{E}+06$ & $1.74 \mathrm{E}+121$ & $3.20 \mathrm{E}+05$ \\
\hline \multirow[t]{3}{*}{ F9 } & minimum & $5.68 \mathrm{E}-14$ & $3.98 \mathrm{E}+01$ & $2.45 \mathrm{E}-07$ & $5.62 \mathrm{E}+00$ & $3.48 \mathrm{E}+01$ \\
\hline & avrage & $1.92 \mathrm{E}+00$ & $6.92 \mathrm{E}+01$ & $1.84 \mathrm{E}+01$ & $7.46 \mathrm{E}+00$ & $5.41 \mathrm{E}+01$ \\
\hline & std & $7.80 \mathrm{E}+00$ & $3.59 \mathrm{E}+02$ & $7.93 \mathrm{E}+02$ & $1.00 \mathrm{E}+00$ & $1.95 \mathrm{E}+02$ \\
\hline \multirow[t]{3}{*}{ F10 } & minimum & $7.55 \mathrm{E}-14$ & $1.16 \mathrm{E}+00$ & $4.44 \mathrm{E}-15$ & $1.20 \mathrm{E}+00$ & $6.58 \mathrm{E}-05$ \\
\hline & avrage & $1.03 \mathrm{E}-13$ & $3.83 \mathrm{E}+00$ & $7.28 \mathrm{E}-15$ & $2.04 \mathrm{E}+00$ & $7.68 \mathrm{E}-05$ \\
\hline & std & $2.35 \mathrm{E}-28$ & $2.11 \mathrm{E}+00$ & $3.52 \mathrm{E}-29$ & $3.47 \mathrm{E}-01$ & $2.88 \mathrm{E}-11$ \\
\hline \multirow[t]{3}{*}{ F11 } & minimum & $0.00 \mathrm{E}+00$ & $7.75 \mathrm{E}+00$ & $0.00 \mathrm{E}+00$ & $1.19 \mathrm{E}+00$ & $1.68 \mathrm{E}-07$ \\
\hline & avrage & $4.80 \mathrm{E}-03$ & $1.54 \mathrm{E}+01$ & $1.13 \mathrm{E}-02$ & $1.92 \mathrm{E}+00$ & $6.08 \mathrm{E}-03$ \\
\hline & std & $6.45 \mathrm{E}-05$ & $2.34 \mathrm{E}+01$ & $3.96 \mathrm{E}-04$ & $1.99 \mathrm{E}-01$ & $5.26 \mathrm{E}-05$ \\
\hline \multirow[t]{3}{*}{ F12 } & minimum & $1.31 \mathrm{E}-02$ & $5.83 \mathrm{E}-01$ & $4.12 \mathrm{E}-02$ & $3.26 \mathrm{E}+05$ & $2.23 \mathrm{E}-10$ \\
\hline & avrage & $4.91 \mathrm{E}-02$ & $3.75 \mathrm{E}+00$ & $1.40 \mathrm{E}+00$ & $6.79 \mathrm{E}+06$ & $1.00 \mathrm{E}-01$ \\
\hline & std & 3.13E-04 & $3.40 \mathrm{E}+00$ & $4.93 \mathrm{E}+00$ & $1.58 \mathrm{E}+13$ & $6.00 \mathrm{E}-02$ \\
\hline \multirow[t]{3}{*}{ F13 } & minimum & $2.32 \mathrm{E}-01$ & $4.05 \mathrm{E}-01$ & $8.00 \mathrm{E}+00$ & $2.92 \mathrm{E}+06$ & 8.65E-08 \\
\hline & avrage & $6.66 \mathrm{E}-01$ & $1.15 \mathrm{E}+02$ & $9.14 \mathrm{E}+00$ & $1.53 \mathrm{E}+07$ & $1.10 \mathrm{E}-07$ \\
\hline & std & $4.02 \mathrm{E}-02$ & $1.02 \mathrm{E}+05$ & $1.86 \mathrm{E}-01$ & $8.48 \mathrm{E}+13$ & $2.24 \mathrm{E}-16$ \\
\hline \multirow[t]{3}{*}{ F14 } & minimum & $3.08 \mathrm{E}-04$ & $3.13 \mathrm{E}-04$ & $3.08 \mathrm{E}-04$ & $8.05 \mathrm{E}-04$ & $3.07 \mathrm{E}-04$ \\
\hline & avrage & $3.10 \mathrm{E}-03$ & $8.34 \mathrm{E}-04$ & 7.31E-04 & $1.05 \mathrm{E}-03$ & $3.77 \mathrm{E}-04$ \\
\hline & std & $4.75 \mathrm{E}-05$ & $1.26 \mathrm{E}-07$ & $1.36 \mathrm{E}-07$ & $5.47 \mathrm{E}-09$ & $2.87 \mathrm{E}-08$ \\
\hline F15 & minimum & $-1.03 \mathrm{E}+00$ & $-1.03 \mathrm{E}+00$ & $-1.03 \mathrm{E}+00$ & $-1.03 \mathrm{E}+00$ & $-1.03 \mathrm{E}+00$ \\
\hline
\end{tabular}




\begin{tabular}{lllllll}
\hline Function & Statitic value & GOW & PSO & HPO & ABC & FA \\
\hline & avrage & $-1.03 \mathrm{E}+00$ & $-1.03 \mathrm{E}+00$ & $-1.03 \mathrm{E}+00$ & $-1.03 \mathrm{E}+00$ & $-1.03 \mathrm{E}+00$ \\
& std & $4.51 \mathrm{E}-16$ & $0.00 \mathrm{E}+00$ & $5.82 \mathrm{E}-21$ & $0.00 \mathrm{E}+00$ & $1.06 \mathrm{E}-28$ \\
\hline
\end{tabular}

Table 4.Result of unimodal and multimodal benchmark function in 100 dimensions

\begin{tabular}{|c|c|c|c|c|c|c|}
\hline Function & Statitic value & GOW & $\mathrm{PSO}$ & HPO & $\mathrm{ABC}$ & FA \\
\hline \multirow[t]{3}{*}{$\overline{\mathrm{F} 1}$} & minimum & $2.49 \mathrm{E}-13$ & $3.98 \mathrm{E}+03$ & $9.23 \mathrm{E}-37$ & $2.41 \mathrm{E}+05$ & $5.47 \mathrm{E}-06$ \\
\hline & avrage & $1.79 \mathrm{E}-12$ & $6.81 \mathrm{E}+03$ & $4.87 \mathrm{E}-31$ & $2.64 \mathrm{E}+05$ & $8.48 \mathrm{E}-06$ \\
\hline & std & $2.97 \mathrm{E}-24$ & $1.70 \mathrm{E}+06$ & $2.04 \mathrm{E}-60$ & $1.50 \mathrm{E}+08$ & $3.03 \mathrm{E}-12$ \\
\hline \multirow[t]{3}{*}{$\mathrm{F} 2$} & minimum & $1.87 \mathrm{E}-08$ & $7.07 \mathrm{E}+01$ & $3.88 \mathrm{E}-19$ & $1.92 \mathrm{E}+26$ & $1.73 \mathrm{E}-03$ \\
\hline & avrage & $3.97 \mathrm{E}-08$ & $1.24 \mathrm{E}+02$ & $1.74 \mathrm{E}-16$ & $7.21 \mathrm{E}+37$ & $1.93 \mathrm{E}-03$ \\
\hline & std & $1.83 \mathrm{E}-16$ & $6.60 \mathrm{E}+02$ & $2.66 \mathrm{E}-31$ & $3.11 \mathrm{E}+76$ & $1.24 \mathrm{E}-08$ \\
\hline \multirow[t]{3}{*}{ F3 } & minimum & $1.35 \mathrm{E}+01$ & $7.63 \mathrm{E}+03$ & $1.14 \mathrm{E}-23$ & $6.27 \mathrm{E}+05$ & $1.16 \mathrm{E}+04$ \\
\hline & avrage & $5.08 \mathrm{E}+02$ & $3.64 \mathrm{E}+04$ & $9.79 \mathrm{E}+01$ & $1.21 \mathrm{E}+06$ & $1.75 \mathrm{E}+04$ \\
\hline & std & $2.67 \mathrm{E}+05$ & $8.48 \mathrm{E}+08$ & $6.18 \mathrm{E}+04$ & $7.58 \mathrm{E}+10$ & $1.44 \mathrm{E}+07$ \\
\hline \multirow[t]{3}{*}{$\mathrm{F} 4$} & minimum & $1.10 \mathrm{E}-01$ & $1.18 \mathrm{E}+01$ & $1.10 \mathrm{E}-15$ & $9.41 \mathrm{E}+01$ & $5.90 \mathrm{E}+01$ \\
\hline & avrage & $8.25 \mathrm{E}-01$ & $1.43 \mathrm{E}+01$ & $1.07 \mathrm{E}-05$ & $9.64 \mathrm{E}+01$ & $8.09 \mathrm{E}+01$ \\
\hline & std & $6.04 \mathrm{E}-01$ & $4.52 \mathrm{E}+00$ & $3.24 \mathrm{E}-09$ & 8.01E-01 & $7.59 \mathrm{E}+01$ \\
\hline \multirow[t]{3}{*}{ F5 } & minimum & $9.62 \mathrm{E}+01$ & $5.79 \mathrm{E}+04$ & $9.70 \mathrm{E}+01$ & $9.82 \mathrm{E}+08$ & $1.74 \mathrm{E}+02$ \\
\hline & avrage & $9.78 \mathrm{E}+01$ & $3.49 \mathrm{E}+06$ & $9.82 \mathrm{E}+01$ & $1.21 \mathrm{E}+09$ & $3.18 \mathrm{E}+02$ \\
\hline & std & $4.48 \mathrm{E}-01$ & $5.01 \mathrm{E}+12$ & $1.91 \mathrm{E}-01$ & $8.66 \mathrm{E}+15$ & $1.19 \mathrm{E}+04$ \\
\hline \multirow[t]{3}{*}{ F6 } & minimum & $8.54 \mathrm{E}+00$ & $3.12 \mathrm{E}+03$ & $1.08 \mathrm{E}+01$ & $2.14 \mathrm{E}+05$ & $5.32 \mathrm{E}-06$ \\
\hline & avrage & $1.01 \mathrm{E}+01$ & $6.71 \mathrm{E}+03$ & $1.34 \mathrm{E}+01$ & $2.62 \mathrm{E}+05$ & $7.94 \mathrm{E}-06$ \\
\hline & std & $7.16 \mathrm{E}-01$ & $2.06 \mathrm{E}+06$ & $1.41 \mathrm{E}+00$ & $3.45 \mathrm{E}+08$ & $2.76 \mathrm{E}-12$ \\
\hline \multirow[t]{3}{*}{ F7 } & minimum & $3.15 \mathrm{E}-03$ & $1.21 \mathrm{E}+03$ & $2.54 \mathrm{E}-04$ & $1.49 \mathrm{E}+03$ & $6.79 \mathrm{E}-02$ \\
\hline & avrage & 7.43E-03 & $1.67 \mathrm{E}+03$ & $1.65 \mathrm{E}-03$ & $1.90 \mathrm{E}+03$ & $1.26 \mathrm{E}-01$ \\
\hline & std & $7.84 \mathrm{E}-06$ & $4.34 \mathrm{E}+04$ & $1.57 \mathrm{E}-06$ & $2.46 \mathrm{E}+04$ & $1.01 \mathrm{E}-03$ \\
\hline \multirow[t]{3}{*}{ F8 } & minimum & $-1.96 \mathrm{E}+04$ & $-8.95 \mathrm{E}+03$ & $-2.65 \mathrm{E}+04$ & $-4.84 \mathrm{E}+61$ & $-2.82 \mathrm{E}+04$ \\
\hline & avrage & $-1.61 \mathrm{E}+04$ & $-5.89 \mathrm{E}+03$ & $-1.90 \mathrm{E}+04$ & $-3.65 \mathrm{E}+60$ & $-2.59 \mathrm{E}+04$ \\
\hline & std & $6.09 \mathrm{E}+06$ & $1.07 \mathrm{E}+06$ & $1.06 \mathrm{E}+07$ & $9.97 \mathrm{E}+121$ & $2.08 \mathrm{E}+06$ \\
\hline \multirow[t]{3}{*}{ F9 } & minimum & $2.97 \mathrm{E}-10$ & $3.68 \mathrm{E}+02$ & $0.00 \mathrm{E}+00$ & $1.41 \mathrm{E}+03$ & $9.56 \mathrm{E}-01$ \\
\hline & avrage & $1.22 \mathrm{E}+01$ & $5.24 \mathrm{E}+02$ & $2.37 \mathrm{E}-01$ & $1.62 \mathrm{E}+03$ & $1.95 \mathrm{E}+00$ \\
\hline & std & $5.48 \mathrm{E}+01$ & $6.03 \mathrm{E}+03$ & $7.12 \mathrm{E}-01$ & $2.60 \mathrm{E}+03$ & $9.65 \mathrm{E}-02$ \\
\hline \multirow[t]{3}{*}{ F10 } & minimum & $5.40 \mathrm{E}-08$ & $6.55 \mathrm{E}+00$ & $4.44 \mathrm{E}-15$ & $2.08 \mathrm{E}+01$ & $9.56 \mathrm{E}-01$ \\
\hline & avrage & $1.13 \mathrm{E}-07$ & $9.15 \mathrm{E}+00$ & $1.34 \mathrm{E}-14$ & $2.09 \mathrm{E}+01$ & $1.95 \mathrm{E}+00$ \\
\hline & std & $1.30 \mathrm{E}-15$ & $2.20 \mathrm{E}+00$ & $2.83 \mathrm{E}-28$ & $3.34 \mathrm{E}-03$ & $9.65 \mathrm{E}-02$ \\
\hline \multirow[t]{3}{*}{ F11 } & minimum & $1.41 \mathrm{E}-13$ & $3.61 \mathrm{E}+01$ & $0.00 \mathrm{E}+00$ & $2.18 \mathrm{E}+03$ & $6.15 \mathrm{E}-06$ \\
\hline & avrage & $4.42 \mathrm{E}-03$ & $7.05 \mathrm{E}+01$ & $1.82 \mathrm{E}-02$ & $2.37 \mathrm{E}+03$ & $1.90 \mathrm{E}-03$ \\
\hline & std & $1.15 \mathrm{E}-04$ & $1.83 \mathrm{E}+02$ & $1.89 \mathrm{E}-03$ & $1.38 \mathrm{E}+04$ & $1.74 \mathrm{E}-05$ \\
\hline \multirow[t]{3}{*}{ F12 } & minimum & $2.16 \mathrm{E}-01$ & $6.10 \mathrm{E}+00$ & $2.88 \mathrm{E}-01$ & $2.20 \mathrm{E}+09$ & $1.05 \mathrm{E}+00$ \\
\hline & avrage & $2.95 \mathrm{E}-01$ & $7.32 \mathrm{E}+03$ & $4.40 \mathrm{E}-01$ & $2.83 \mathrm{E}+09$ & $4.38 \mathrm{E}+00$ \\
\hline & std & $4.78 \mathrm{E}-03$ & $4.07 \mathrm{E}+08$ & $7.60 \mathrm{E}-03$ & $8.01 \mathrm{E}+16$ & $6.85 \mathrm{E}+00$ \\
\hline \multirow[t]{3}{*}{ F13 } & minimum & $6.07 \mathrm{E}+00$ & $3.24 \mathrm{E}+01$ & $8.42 \mathrm{E}+00$ & $2.20 \mathrm{E}+09$ & $5.03 \mathrm{E}-02$ \\
\hline & avrage & $6.85 \mathrm{E}+00$ & $1.42 \mathrm{E}+06$ & $9.09 \mathrm{E}+00$ & $2.89 \mathrm{E}+09$ & $1.20 \mathrm{E}+01$ \\
\hline & std & $1.69 \mathrm{E}-01$ & $1.87 \mathrm{E}+12$ & $1.29 \mathrm{E}-01$ & $6.12 \mathrm{E}+16$ & $8.96 \mathrm{E}+01$ \\
\hline \multirow[t]{3}{*}{ F14 } & minimum & $3.08 \mathrm{E}-04$ & 3.07E-04 & $3.08 \mathrm{E}-04$ & $5.71 \mathrm{E}-04$ & 3.07E-04 \\
\hline & avrage & $6.42 \mathrm{E}-03$ & $8.48 \mathrm{E}-04$ & 8.07E-04 & $9.69 \mathrm{E}-04$ & $1.09 \mathrm{E}-03$ \\
\hline & std & $8.62 \mathrm{E}-05$ & $6.78 \mathrm{E}-08$ & $1.37 \mathrm{E}-07$ & $1.61 \mathrm{E}-08$ & $3.22 \mathrm{E}-07$ \\
\hline F15 & minimum & $-1.03 \mathrm{E}+00$ & $-1.03 \mathrm{E}+00$ & $-1.03 \mathrm{E}+00$ & $-1.03 \mathrm{E}+00$ & $-1.03 \mathrm{E}+00$ \\
\hline
\end{tabular}




\begin{tabular}{lllllll}
\hline Function & Statitic value & GOW & PSO & HPO & ABC & FA \\
\hline & avrage & $-1.03 \mathrm{E}+00$ & $-1.03 \mathrm{E}+00$ & $-1.03 \mathrm{E}+00$ & $-5.15 \mathrm{E}-01$ & $-1.03 \mathrm{E}+00$ \\
& std & $3.07 \mathrm{E}-16$ & $0.00 \mathrm{E}+00$ & $6.36 \mathrm{E}-21$ & $2.76 \mathrm{E}-01$ & $1.27 \mathrm{E}-28$ \\
\hline
\end{tabular}

Table 5. Wilcoxon rank test in 30 dimensions

\begin{tabular}{lllllllll}
\hline Function & GOW $(p)$ & $\mathrm{h}$ & $\mathrm{PSO}(\mathrm{p})$ & $\mathrm{h}$ & $\mathrm{ABC}(\mathrm{p})$ & $\mathrm{h}$ & $\mathrm{FA}(\mathrm{p})$ & $\mathrm{h}$ \\
\hline F1 & $1.73 \mathrm{E}-06$ & 1 & $1.73 \mathrm{E}-06$ & 1 & $1.73 \mathrm{E}-06$ & 1 & $1.72 \mathrm{E}-06$ & 1 \\
F2 & $1.73 \mathrm{E}-06$ & 1 & $1.73 \mathrm{E}-06$ & 1 & $1.73 \mathrm{E}-06$ & 1 & $1.73 \mathrm{E}-06$ & 1 \\
F3 & $1.73 \mathrm{E}-06$ & 1 & $1.73 \mathrm{E}-06$ & 1 & $1.73 \mathrm{E}-06$ & 1 & $1.73 \mathrm{E}-06$ & 1 \\
F4 & $1.73 \mathrm{E}-06$ & 1 & $1.73 \mathrm{E}-06$ & 1 & $1.73 \mathrm{E}-06$ & 1 & $1.73 \mathrm{E}-06$ & 1 \\
F5 & 0.0078 & 1 & $1.73 \mathrm{E}-06$ & 1 & $1.73 \mathrm{E}-06$ & 1 & 0.023 & 1 \\
F6 & $1.48 \mathrm{E}-04$ & 1 & $1.73 \mathrm{E}-06$ & 1 & $1.73 \mathrm{E}-06$ & 1 & $1.73 \mathrm{E}-06$ & 1 \\
F7 & 0.0316 & 1 & $1.73 \mathrm{E}-06$ & 1 & $1.73 \mathrm{E}-06$ & 1 & $1.92 \mathrm{E}-06$ & 1 \\
F8 & 0.0018 & 1 & $1.73 \mathrm{E}-06$ & 1 & $1.73 \mathrm{E}-06$ & 1 & 0.0068 & 1 \\
F9 & 0.0039 & 1 & $6.34 \mathrm{E}-06$ & 1 & 0.8936 & 1 & $1.97 \mathrm{E}-05$ & 1 \\
F10 & $1.69 \mathrm{E}-06$ & 1 & $1.73 \mathrm{E}-06$ & 1 & $1.73 \mathrm{E}-06$ & 1 & $1.73 \mathrm{E}-06$ & 1 \\
F11 & 0.2097 & 0 & $1.73 \mathrm{E}-06$ & 1 & $1.73 \mathrm{E}-06$ & 1 & 0.9426 & 0 \\
F12 & $3.88 \mathrm{E}-06$ & 1 & $4.07 \mathrm{E}-05$ & 1 & $1.73 \mathrm{E}-06$ & 1 & $4.20 \mathrm{E}-04$ & 1 \\
F13 & $1.73 \mathrm{E}-06$ & 1 & 0.9918 & 0 & $1.73 \mathrm{E}-06$ & 1 & $1.73 \mathrm{E}-06$ & 1 \\
F14 & 0.2712 & 0 & 0.2623 & 0 & $1.06 \mathrm{E}-04$ & 1 & $1.89 \mathrm{E}-04$ & 1 \\
F15 & 1 & 0 & $4.32 \mathrm{E}-08$ & 1 & $4.32 \mathrm{E}-08$ & 1 & $4.32 \mathrm{E}-08$ & 1 \\
\hline
\end{tabular}

Table 6. Wilcoxon rank test on 100 dimensions

\begin{tabular}{lllllllll}
\hline Function & GOW $(p)$ & $\mathrm{h}$ & $\mathrm{PSO}(\mathrm{p})$ & $\mathrm{h}$ & $\mathrm{ABC}(\mathrm{p})$ & $\mathrm{h}$ & $\mathrm{FA}(\mathrm{p})$ & $\mathrm{h}$ \\
\hline F1 & $1.73 \mathrm{E}-06$ & 1 & $1.73 \mathrm{E}-06$ & 1 & $1.72 \mathrm{E}-06$ & 1 & $1.73 \mathrm{E}-06$ & 1 \\
F2 & $1.73 \mathrm{E}-06$ & 1 & $1.73 \mathrm{E}-06$ & 1 & $1.73 \mathrm{E}-06$ & 1 & $1.73 \mathrm{E}-06$ & 1 \\
F3 & $9.71 \mathrm{E}-05$ & 1 & $1.73 \mathrm{E}-06$ & 1 & $1.73 \mathrm{E}-06$ & 1 & $1.73 \mathrm{E}-06$ & 1 \\
F4 & $1.73 \mathrm{E}-06$ & 1 & $1.73 \mathrm{E}-06$ & 1 & $1.73 \mathrm{E}-06$ & 1 & $1.73 \mathrm{E}-06$ & 1 \\
F5 & 0.0044 & 1 & $1.73 \mathrm{E}-06$ & 1 & $1.73 \mathrm{E}-06$ & 1 & $1.73 \mathrm{E}-06$ & 1 \\
F6 & $1.73 \mathrm{E}-06$ & 1 & $1.73 \mathrm{E}-06$ & 1 & $1.73 \mathrm{E}-06$ & 1 & $1.73 \mathrm{E}-06$ & 1 \\
F7 & $1.73 \mathrm{E}-06$ & 1 & $1.73 \mathrm{E}-06$ & 1 & $1.73 \mathrm{E}-06$ & 1 & $1.73 \mathrm{E}-06$ & 1 \\
F8 & $5.48 \mathrm{E}-04$ & 1 & $1.73 \mathrm{E}-06$ & 1 & $1.73 \mathrm{E}-06$ & 1 & $1.73 \mathrm{E}-06$ & 1 \\
F9 & $2.88 \mathrm{E}-06$ & 1 & $1.73 \mathrm{E}-06$ & 1 & $1.73 \mathrm{E}-06$ & 1 & $3.41 \mathrm{E}-05$ & 1 \\
F10 & $1.73 \mathrm{E}-06$ & 1 & $1.73 \mathrm{E}-06$ & 1 & $1.73 \mathrm{E}-06$ & 1 & $1.73 \mathrm{E}-06$ & 1 \\
F11 & 0.4908 & 0 & $1.73 \mathrm{E}-06$ & 1 & $1.73 \mathrm{E}-06$ & 1 & 0.382 & 0 \\
F12 & $2.35 \mathrm{E}-06$ & 1 & $1.73 \mathrm{E}-06$ & 1 & $1.73 \mathrm{E}-06$ & 1 & $1.73 \mathrm{E}-06$ & 1 \\
F13 & $1.73 \mathrm{E}-06$ & 1 & $1.73 \mathrm{E}-06$ & 1 & $1.73 \mathrm{E}-06$ & 1 & 0.221 & 0 \\
F14 & 0.544 & 0 & 0.6884 & 0 & 0.0016 & 1 & 0.0387 & 1 \\
F15 & 1 & 0 & $4.32 \mathrm{E}-08$ & 1 & 0.0188 & 1 & 1 & 0 \\
\hline
\end{tabular}




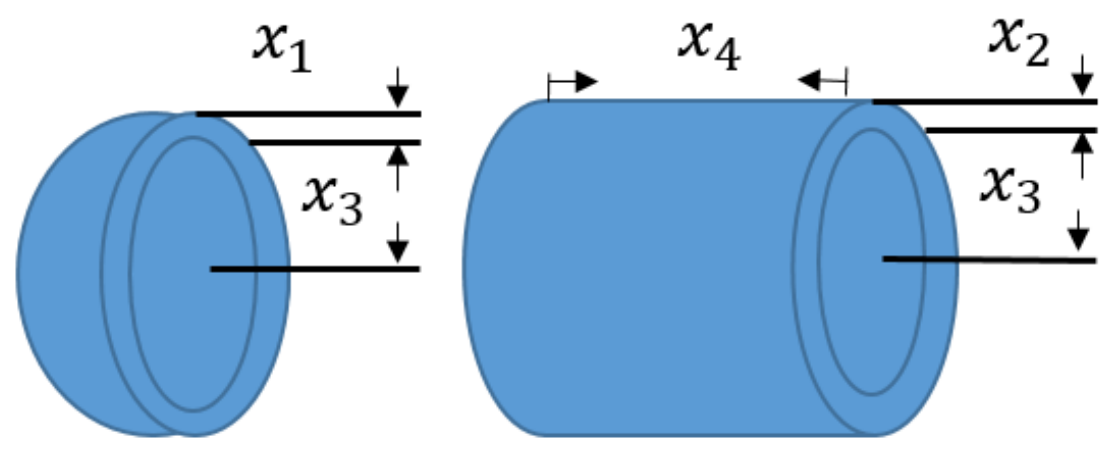

Fig 8. Vessel Pressure

Table 7.comparing all algorithms in vessel pressure design optimization

\begin{tabular}{lllll}
\hline Algorithm & Optimum Variables & Optimum Variables & Optimum Variables & Optimum Variables \\
\hline & $T_{s} T_{h} \mathrm{R} \mathrm{L}$ & $T_{s} T_{h} \mathrm{R} \mathrm{L}$ & $T_{s} T_{h} \mathrm{R} \mathrm{L}$ & $T_{s} T_{h} \mathrm{R} \mathrm{L}$ \\
HPO & $\mathbf{0 . 7 5 7 9 2 5 1 9 3 4 6 2 1 6 8}$ & $\mathbf{0 . 3 7 4 6 4 2 8 1 5 8 3 6 1 3 8}$ & $\mathbf{3 9 . 2 7 0 7 3 5 4 1 2 7 4 1 9}$ & $\mathbf{2 1 5 . 1 3 4 8 7 6 6 3 0 0 6 7}$ \\
GWO(SeyedaliMirjaliliet al.) & 0.812500 & 0.434500 & 42.089181 & 176.758731 \\
GA (Coelloet al.) & 0.812500 & 0.437500 & 42.097398 & 176.654050 \\
PSO (He et al.) & 0.812500 & 0.437500 & 42.091266 & 176.746500 \\
ABC (B.Akay et al.) & 0.812500 & 0.437500 & 42.098446 & 176.636596 \\
\hline
\end{tabular}

\section{CONCLUSION}

This study provided a novel algorithm that arises from happiness behavior of personal in workplace. Three criteria aredefined for whole search space, it was adjustable approach to less and more dimensions. The experiment result with statistical values and Wilcoxon rank test showed HPO algorithm has more reliability, robustness, flexible and stability than the other algorithms. This workfocused to provide balancing between exploration and exploitation with tuning damping operators and mentioned criterions, as well as, covering the different search space.For future work, we are planning to adapt our work with neural network and fuzzy systems such as multilayer perceptron and adaptive-network-based fuzzy inference system(ANFIS)design that in order to adjust the weight parameters. They areknowledge of system, which provide classification, clustering and estimation task. An another hand, by improving our method we can propose a multi-objectivealgorithm for complex real problem.

[1] Blum C, Puchinger J, Raidl GR, Roli A. Hybrid metaheuristics in ombinatorial. optimization: a survey. Appl Soft Comput 2011;11:4135-51.

[2] Boussaïd I, Lepagnot J, Siarry P. A survey on optimization metaheuristics.

Inform Sci 2013;237:82-117.

[3] Gogna A, Tayal A. Metaheuristics: review and application. J ExpTheorArtif

[4] Lazar A (2002) Heuristic knowledge discovery for archaeological data using genetic algorithms and rough sets. In: Sarker R, Abbass H, Newton C (eds) Heuristic and optimization for knowledge discovery. IGI Global, Hershey, pp 263-278.

[5] X-.-S. Yang, Nature-Inspired Optimization Algorithms, ElsevierInsight,London, 2014

[6]Holland J (1975) Adaptation in natural and artificial systems: anintroductory analysis with application to biology. Control andartificial intelligence. MIT press, Cambridge. 
[7]D.E. Goldberg, Genetic Algorithms in Search, Optimization and MachineLearning, Addison-Wesley, Reading, MA, USA, 1989.

[8] Wolpert DH, Macready WG. No free lunch theorems for optimization. Evolut

Comput, IEEE Trans 1997;1:67-82.

[9] Glover F (1986) Future paths for integer programming and links to artificial intelligence. ComputOper Res 13(5):533-549.https://doi.org/10.1016/0305-0548(86)90048-1

[10] Van Laarhoven PJ, Aarts EH (1987) Simulated annealing. In:Aart EH, van Laarhoven PJ (eds) Simulated annealing: theoryand applications. Springer, Berlin, pp 7-15

[11] Ghaemi M, Feizi-Derakhshi M-R (2014) Forest optimizationalgorithm. Expert SystAppl 41(15):66766687. https://doi.org/10.1016/j.eswa.2014.05.009

[12] Simon D (2008) Biogeography-based optimization. IEEE TransEvolComput 12(6):702-713. https://doi.org/10.1109/TEVC. 2008.919004

[13] Yao X, Liu Y, Lin G. Evolutionary programming made faster. EvolutComput,

IEEE Trans 1999;3:82-102.

[14] Hansen N, Müller SD, Koumoutsakos P. Reducing the time complexity of the

derandomized evolution strategy with covariance matrix adaptation (CMAES).

EvolutComput 2003;11:1-18.

[15] Beni G, Wang J. Swarm intelligence in cellular robotic systems. In: Robots and

biological systems: towards a new bionics?, ed. Springer; 1993. p. 703-12.

[16] Eberhart R, Kennedy JA (1995) New optimizer using particleswarm theory. In: MHS'95. proceedings of the sixth internationalsymposium on micro machine and human science, 4-6 Oct. 1995. pp 39-43. https://doi.org/10.1109/mhs.1995.494215

[17] X. Li, P. Tian, X. Min, "A Hierarchical Particle Swarm Optimization for Solving Bilevel Programming Problems," Lecture Notes in Computer Science , 4029, pp.1169-1178, 2006.

[18] Hamed Shah-Hosseini," Intelligent water drops algorithm: A new optimization method for solving the multiple knapsack problem", International Journal of Intelligent Computing and Cybernetics, Vol. 1 Issue: 2, pp.193-212, 2008

[19] Chu S-C, Tsai P-w, Pan J-S (2006) Cat swarm optimization. In:Yang Q, Webb G (eds) PRICAI 2006: trends in artificial intelligence.Springer, Berlin, pp 854-858

[20]Karaboga D, Basturk B (2007) A powerful and efficient algorithmfor numerical function optimization: artificial bee colony (ABC)algorithm. J Global Optim 39(3):459-471. https://doi.org/10.1007/s10898-0079149-x

[21]EsmatRashedi, Hossein Nezamabadi-pour, SaeidSaryazdi, "GSA: A Gravitational Search Algorithm", Information Sciences, Volume 179, Issue 13, 2009, Pages 2232-2248,https://doi.org/10.1016/j.ins.2009.03.004.

[22] Fausto F, Cuevas E, Valdivia A, Gonza'lez A (2017) A globaloptimization algorithm inspired in the behavior of selfish herds.Biosystems 160:39-55. https://doi.org/10.1016/j.biosystems.2017.07.010

[23] A. Kaveh, N. Farhoudi, A new optimization method: Dolphin echolocation, Adv.Eng. Softw. 59 (2013) $53-70$.

[24]R. A. Formato, "Central force optimization: a new metaheuristic with applications in applied electromagnetics," Progress in Electromagnetics Research, vol. 77, pp. 425-491, 2007. 
[25]L. Xie, J. Zeng, and Z. Cui, "General framework of artificial physics optimization algorithm," in Proceedings of the World Congress on Nature and Biologically Inspired Computing (NaBIC '09), pp. 1321-1326, IEEE, December 2009.

[26]E. Rashedi, H. Nezamabadi-Pour, and S. Saryazdi, "GSA: a gravitational search algorithm," Information Sciences, vol. 179, no. 13, pp. 2232-2248, 2009.

[27] J. Flores, R. Lopez, and J. Barrera, "Gravitational interactions ' optimization," in Learning and Intelligent Optimization, pp. 226-237, Springer, Berlin, Germany, 2011

[28]Wolpert DH, Macready WG. No free lunch theorems for optimization. EvolutComput, IEEE Trans 1997;1:67-82.

[29] May 24, 2012, Q\&A with the authors of Engineering Happiness: "A new approach for building a joyful life" Engineering Happiness: "a new approach for building a joyful life".

[30] De Juan, R., Mochón, F., Rojas, M.: Expectations and happiness: evidence from Spain. J. Soc. Res. Policy 5(2), 101-114 (2014).

[31]https://medium.com/@h_liyan/an-equation-for-happiness-71f5b9a22836 